Schließlich wurde der Grenzfall einer nicht kontinuierlich verteilten, sondern punktförmig an einer Stelle angreifenden Kraft untersucht ${ }^{*}$. Durch Änderung der Frequenz der anregenden Kraft erhält man alle Eigenfrequenzen als Resonanzen eines Stabes, außer denen, in deren Eigenschwingungsknoten die Kraft angreift (Abb. 8, 9, 10). Die hier- bei gemessenen Resonanzamplituden sind aber mit einem Faktor bewertet, der aus Stablänge und relativem Abstand des Angriffspunktes zum nächsten Knoten der jeweiligen Eigenschwingungsform gebildet wird.

* Dieser Fall wurde für den Stab ohne Dämpfung mit konstantem Querschnitt von Timoschenко ${ }^{4}$ untersucht.

\title{
Das Verhalten von p-n-Gleichrichtern bei hohen Durchlaßbelastungen
}

\author{
Von A. Herlet \\ Aus dem Laboratorium der Siemens-Schuckert-Werke AG., Pretzfeld \\ (Z. Naturforschg. 11 a, 498-510 [1956]; eingegangen am 19. April 1956) \\ Herrn Professor Trendelenburg zum 60. Geburtstag gewidmet
}

\begin{abstract}
Das Verhalten eines modellmäßig vereinfachten p-n-Gleichrichters bei hohen Durchlaßströmen, d. h. außerhalb des Gültigkeitsbereiches der Sноскцегschen Theorie, wird untersucht. Es wird wesentlich beherrscht von einem „unbegrenzten“ Anwachsen beider Trägerkonzentrationen mit $V \bar{i}$ über die Dotierungskonzentration hinaus. Der Spannungsabfall über den Bahngebieten wächst daher nur mit $\sqrt{i}$, während der Spannungsabfall über dem Raumladungsgebiet erwartungsgemäß den Grenzwert $V_{\mathrm{D}}$ anstrebt. Man erhält daher eine quadratische Strom-Spannungs-Charakteristik: $i \sim\left(U-V_{\mathrm{D}}\right)^{2}$.
\end{abstract}

Die Sноскцеysche Theorie ${ }^{1}$ liefert als Kennliniengleichung eines p-n-Gleichrichters die Beziehung

$i=e\left\{p_{\mathrm{n}} \frac{D_{\mathrm{p}}}{L_{\mathrm{p}}}+n_{\mathrm{p}} \frac{D_{\mathrm{n}}}{L_{\mathrm{n}}}\right\}\left(e^{U / \mathfrak{B}}-1\right) ; \quad \mathfrak{V}=\frac{k T}{e}$.

Die Gültigkeit von (1) ist an die Voraussetzung niedriger Injektion gebunden, d. h. die Konzentrationen der in die Bahngebiete injizierten Minoritätsträger müssen klein gegen die Majoritätsträgerkonzentration bzw. die Dotierungskonzentration sein. Im Gültigkeitsbereich dieser SноскLEyschen Näherung wird daher die geforderte elektrische Neutralität in den Bahngebieten durch die Beziehungen

$$
\begin{array}{ll}
p=n_{\mathrm{A}^{-}}, & x \leqq x_{\mathrm{p}}, \\
n=n_{\mathrm{D}^{+}}, & x \geqq x_{\mathrm{n}}
\end{array}
$$

gewahrt (Abb. 1) ; die Konzentrationen der Majoritätsträger bleiben auch im Belastungsfall praktisch unverändert. Ferner ist im Bereich der SноcкLEyschen Näherung der Spannungsabfall über den Bahngebieten bei vernünftiger geometrischer Abmessung

1 W. Shockley, Bell Syst. Techn. J. 28, 435 [1949].

2 Streng genommen bedeutet die Spannung $U$ in (1) den Spannungsabfall über der Raumladungszone. Die vollständige Kennlinie erhält man bei niedrigen Injektionen durch Scherung von (1) mit dem Widerstand der Bahngebiete vernachlässigbar ${ }^{2}$. Die gesamte äußere Spannung wird von der Raumladungszone zwischen $x_{\mathrm{p}}$ und $x_{\mathrm{n}}$ aufgenommen und vermindert die dort im stromlosen Zustand vorhandene Diffusionsspannung $V_{\mathrm{D}}$ (Abb. 1).

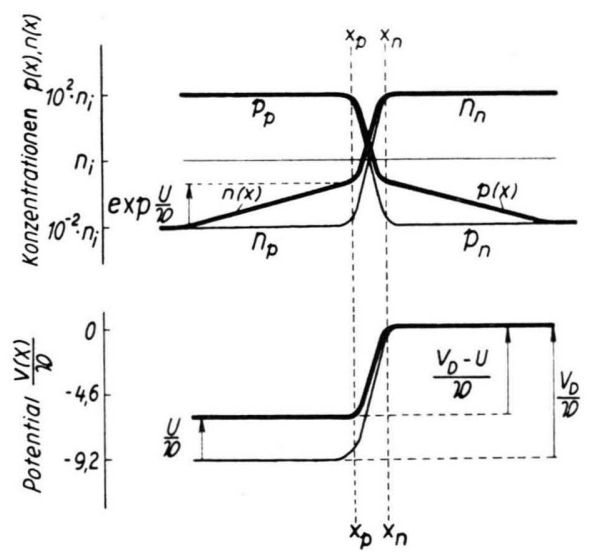

Abb. 1. Konzentrationsverteilungen und Potentialverlauf in einem p-n-Gleichrichter bei niedrigen Injektionen (Shockleysche Theorie).

(vgl. ${ }^{3}$, S. 78). Freilich wird der Einfluß des Bahnwiderstandes erst stark bemerkbar, wenn die Injektion nicht mehr niedrig ist, und dann gilt (1) nicht mehr.

${ }^{3}$ E. SPEnke, Elektronische Halbleiter, Springer-Verlag 1955. 
Steigert man nun die äußere Spannung immer weiter, so muß zweifellos dieser geschilderte Mechanismus versagen und man wird etwa folgendes Verhalten des p-n-Gleichrichters erwarten: Die Diffusionsspannung $V_{\mathrm{D}}$ ist abgebaut, die Raumladungszone ist zugeweht und kann über den Wert von $V_{\mathrm{D}}$ hinaus keinen zusätzlichen Anteil der äußeren Spannung mehr aufnehmen. Diese wird vielmehr jetzt als Spannungsabfall über den „Онмschen“ Bahngebieten verbraucht; man wird also einen quasi-Oнмschen Kennlinienverlauf

$$
i={ }_{F R_{\mathrm{b}}}^{1} \cdot\left(U-V_{\mathrm{D}}\right)
$$

erwarten, wobei jetzt die Frage auftaucht, wie sich der in (4) auftretende "Bahnwiderstand“ $R_{\mathrm{l}}$, bestimmt.

Man wird hier zunächst geneigt sein, für die Berechnung des „Bahnwiderstandes" die durch die Dotierung gegebenen spezifischen Widerstände des n- und p-Gebietes einzusetzen, d. h. also mit einer durch (2) bzw. (3) gegebenen Konzentration von Stromträgern zu rechnen. Diese Annahme ist aber nun keineswegs zwingend. Mit wachsender Spannung wird ja - zunächst im Gültigkeitsbereich der Shockleyschen Theorie - die Konzentration der Minoritätsträger dauernd angehoben, so daß diese schließlich bei völligem Abbau der Diffusionsspannung $V_{\mathrm{D}}$ in die Größenordnung der Majoritätsträgerkonzentration kommen müssen. Dann können die Minoritätsträger aber in der Raumladungsbilanz nicht mehr vernachlässigt werden und die Gln. (2) und (3) müssen durch die Beziehungen

$$
\begin{array}{ll}
p=n_{\mathrm{A}^{-}}+n, & x \leqq x_{\mathrm{p}}, \\
n=n_{\mathrm{D}^{+}}+p, & x \geqq x_{\mathrm{D}}
\end{array}
$$

ersetzt werden. Das Anheben der Minoritätsträgerkonzentrationen, also z. B. der Elektronen im p-Gebiet, muß nach (5) und (6) in den Bahngebieten ein gleich starkes Anwachsen der Majoritätsträgerkonzentrationen, z. B. der Defektelektronen im pGebiet, nach sich ziehen. Infolge dieser angehobenen Majoritätsträgerkonzentration ist aber bei einer weiteren Steigerung der Stromdichte bzw. der äußeren Spannung wieder eine Steigerung der Injektion und damit eine Erhöhung der Minoritätsträgerkonzentrationen möglich.

Bei welchen Konzentrationswerten kommt dieses gegenseitige Emportreiben von Majoritäts- und Minoritätsträgerkonzentrationen zum Stillstand? In Abschnitt I werden wir zeigen, daß die Konzentra- tionsanhebung in den Bahngebieten gar nicht zum Stillstand kommt, sondern „beliebig“ weitergeht, so daß man in den Bahngebieten eine ständig wachsende Leitfähigkeit zur Verfügung hat. Das führt an Stelle von (4) zu einer im wesentlichen quadratischen Strom-Spannungs-Beziehung, die wir in Abschnitt III diskutieren wollen. Vor der Kennliniendiskussion wollen wir jedoch in Abschnitt II das Raumladungsgebiet behandeln. Die oben erwähnten qualitativen Vorstellungen werden dabei quantitativ bestätigt bzw. konkretisiert.

Aus dieser Inhaltsübersicht geht schon hervor, daß wir die bei niedrigen Injektionen (SHockleysche Theorie) durchgeführte räumliche Auftrennung des p-n-Gleichrichters in eine Raumladungszone und 2 Bahngebiete beibehalten werden. Im Falle hoher Durchlaßbelastungen werden jedoch diese Bahngebiete, die bei niedrigen Injektionen mehr oder weniger bedeutungslos waren, wichtig, so daß wir uns wenigstens im Prinzip mit einer Festlegung der Länge und der Begrenzung dieser Bahngebiete, also mit der Frage der Kontaktierung befassen müssen. Da diese Gesichtspunkte jedoch auf die prinzipiellen Ergebnisse ohne Einfluß sind, werden wir uns mit recht vereinfachenden Annahmen begnügen.

Auch sonst wollen wir unsere Betrachtungen nach Möglichkeit vereinfachen. Wir werden daher mit einem völlig symmetrischen p-n-Gleichrichter rechnen, d. h. mit
a) gleich hoher Dotierungskonzentration im p- und n-Gebiet,
b) abruptem Störstellenverlauf,
c) gleicher Beweglichkeit von Elektronen und Defektelektronen,
d) gleicher Diffusionslänge im p- und n-Gebiet.

Mit diesen Vereinfachungen nimmt die SHockLeysche Kennliniengleichung (1), auf die wir noch einmal kurz zurückkommen wollen, die Form

$$
i=2 e(D / L) n_{\mathrm{p}}\left(e^{U / \mathfrak{B}}-1\right) \approx 2 e(D / L) n_{\mathrm{p}} e^{U / \mathfrak{B}}
$$

an. Der Faktor $n_{\mathrm{p}} e^{U / \mathfrak{B}}$ ist dabei die Randkonzentration der Minoritätsträger am Ende der Raumladungszone bei $x_{\mathrm{p}}$ bzw. bei $x_{\mathrm{n}}$ (vgl. $\left.{ }^{3}, \mathrm{~S} .99\right)$. Wir haben weiter oben dargestellt, daß die Voraussetzungen der Shockleyschen Theorie nicht mehr erfüllt sind, wenn diese Randkonzentrationen in die Größenordnung der Dotierungskonzentrationen $n_{A^{-}}$bzw. $n_{D^{+}}$ kommen. Man wird daher geneigt sein, den Schnitt zwischen der SHockLeyschen Näherung und einer 
Näherungslösung für hohe Durchlaßbelastungen bei einer Stromdichte

$$
2 e(D / L) n_{\mathrm{A}^{-}}
$$

zu erwarten, bei der also die Randkonzentration der Minoritätsträger bei formaler Zugrundelegung der Shockleyschen Theorie gerade gleich der Dotierungskonzentration im p- und n-Gebiet geworden wäre. Diese Orientierung ist für uns insofern von Bedeutung, als wir bei der Behandlung der hohen Durchlaßbelastung nicht mehr wie in der SноскLEyschen Theorie die äußere Spannung als vorgegebenen Parameter benutzen wollen, sondern die Stromdichte $i$.

\section{Die Bahngebiete}

Das Geschehen in den Bahngebieten wird im hohen Maße durch die Quasineutralitätsbedingungen (5) und (6) beherrscht, die auch bei hohen Stromdichten noch gut erfüllt sind ${ }^{4}$. Aus ihnen folgt zunächst einmal die Gleichheit der Konzentrationsgradienten von Elektronen und Defektelektronen:

$$
\mathrm{d} p / \mathrm{d} x=\mathrm{d} n / \mathrm{d} x .
$$

Damit wird auf Grund unserer Voraussetzung völliger Symmetrie die Gesamtstromdichte durch die Beziehung

$$
\begin{aligned}
i & =e D\left\{-\frac{\mathrm{d} p}{\mathrm{~d} x}+\frac{\mathrm{d} n}{\mathrm{~d} x}\right\}+e \mu(p+n) E \\
& =e \mu(p+n)
\end{aligned}
$$

gegeben, die für das p-Gebiet, auf das wir uns im folgenden beschränken wollen, mit (5) folgende Form annimmt:

$$
i=e \mu\left(2 p-n_{\mathrm{A}^{-}}\right) \text {E. }
$$

Damit haben wir eine Beziehung für die Bestimmung der beiden Unbekannten $p(x)$ und $(E(x)$ gewonnen. Die zweite Beziehung erhalten wir aus der Kontinuitätsgleichung ${ }^{7}$

$$
\mathrm{d} i_{\mathrm{p}} / \mathrm{d} x=-e \Re,
$$

${ }^{4}$ Der Zwang zur Quasineutralität ist in größeren homogenen Halbleiterbereichen außerordentlich stark, da schon minimale Raumladungsdichten in Bereichen von der Bahnlänge, also z. B. von 0,1 bis $1 \mathrm{~mm}$ genügen, um enorme Potentialunterschiede aufzubauen. Raumladungsgebiete sind daher im allgemeinen auf Bereiche in der Größenordnung der DeBye-Länge, also von maximal einigen $\mu$, begrenzt. (Man vgl. Abschnitt II und ${ }^{5}$, S. 106, S. 153 und S. 161.) Eine Ausnahme bilden lediglich p-s-n-Gleichrichter mit sehr hoher Sperrbelastung und zugleich sehr niedriger Dotierung der Mittelzone, also großer DeBYe-Länge (s. ${ }^{6}$, die die Divergenz des Defektelektronenstromes mit dem Rekombinationsüberschuß $\Re$ verbindet. Die Auswertung dieser Gleichung führt im allgemeinen auf recht komplizierte Differentialgleichungen für die Konzentrationsverteilungen im Bahngebiet. Gerade den technisch interessanten Fall jedoch kann man durch einen Näherungsansatz beschreiben, der zu einer sehr einfachen Darstellung führt. Dieser einfache Sonderfall ist der Fall „kurzer“ Bahngebiete.

Wir wollen die Bahngebiete dann als „kurz“ bezeichnen, wenn sich die Zusammensetzung des Gesamtstromes aus Elektronenstrom und Defektelektronenstrom in den Bahngebieten, d. h. also praktisch von einer Elektrode bis zur andern, nicht wesentlich ändert. Unter der Voraussetzung völliger Symmetrie bedeutet das, daß im ganzen Gleichrichter unabhängig vom Ort der Strom praktisch zur Hälfte als Elektronenstrom, zur Hälfte als Defektelektronenstrom geführt werden soll ${ }^{8}$ (Abb. 2). Die Erzeugung

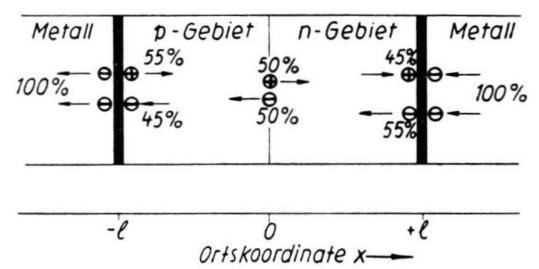

Abb. 2. p-n-Gleichrichter mit „kurzen“ Bahngebieten und „sperrfreien“ Metallkontaktierungen.

der notwendigen Defektelektronen am linken Ende und ihre Vernichtung am rechten Ende des Gleichrichters soll "sperrfreien“ und mit unendlicher Rekombinationsfähigkeit ausgestatteten Grenzflächen vorbehalten sein, die wir uns z. B. durch eine Metallkontaktierung hergestellt denken ${ }^{9}$. Um einen möglichst reinen Fall herauszupräparieren und unnötige Komplikationen zu vermeiden, denken wir uns ferner diese Metallkontaktierungen so vorgenommen, $\mathrm{da} ß$ an der Metall - Halbleiter-Grenze keine Raumladungsrandschichten entstehen. Diese werden nur

Fußnote 6). Man vgl. ferner zu diesen Betrachtungen den Text am Schluß dieses Abschnitts.

5 A. Herlet u. E. Spenke, Z. angew. Phys. 7, 99, 149, 195 [1955].

6 A. Herlet u. H. Patalong, Z. Naturforschg. 10 a, 584 [1955].

7 Man vgl. ${ }^{5}$, S. 100.

8 Von der Brauchbarkeit dieser Näherungsannahme werden wir uns später überzeugen [vgl. Text nach Gl. (28a)].

9 Vgl. hierzu auch die Arbeit von E. SPENKE, Z. Naturforschg. 11 a, 440 [1956]. 
dann vermieden, wenn die durch das Metall erzwungenen, belastungsunabhängigen Randkonzentrationen von Elektronen und Defektelektronen gerade gleich den Gleichgewichtskonzentrationen im Halbleiterinnern sind, wenn also

$$
\begin{aligned}
& p(-l)=p_{\mathrm{p}} \approx n_{\mathrm{A}^{-}}, \\
& n(+l)=n_{\mathrm{n}} \approx n_{\mathrm{D}^{+}}
\end{aligned}
$$

gilt, und zwar für alle Belastungen.

Die SноскLEysche Theorie macht folgende beiden Voraussetzungen: „kleine Injektionen“ und „unendlich ausgedehnte Bahngebiete“. Das Fallenlassen der zweiten Voraussetzung ist sehr einfach, wenn die Metallkontaktierungen bei $x= \pm l$ als sperrfrei vorausgesetzt werden, wenn also die Randbedingungen (13) und (14) akzeptiert werden. Dann erhält man statt des exponentiellen Abklingens der Konzentrationserhöhung in einer unendlich ausgedehnten Bahn einen steileren Abfall der Minoritätsträgerkonzentration, so daß die Stromdichte gegenüber (7) steigt, und zwar um den Faktor $\operatorname{Cotg}(l / L)$ :

$$
i=2 e(D / L) \operatorname{Cotg}(l / L) n_{\mathrm{p}}\left(e^{U / \mathfrak{B}}-1\right) .
$$

Für große Bahnlängen $l \gg L$ geht (15) in (7) über, während sich für $l \ll L$ die Beziehung

$$
i=2 e(D / l) n_{\mathrm{p}}\left(e^{U / \mathfrak{B}}-1\right)
$$

ergibt. Anschaulich kommt (16) dadurch zustande, daß bei kurzen Bahngebieten die Elektronenkonzentration von ihrem Randwert $n_{\mathrm{p}} e^{U / \mathfrak{B}}$ innerhalb der Bahnlänge $l$ linear auf ihren (vergleichsweise verschwindenden) Gleichgewichtswert $n_{\mathrm{p}}$ abfällt.

(15) und (16) sind immer noch an die erste Voraussetzung (kleine Injektion) gebunden. Sie gelten also nur für $n_{\mathrm{p}} e^{U / \mathfrak{B}}<n_{\mathrm{A}^{-}}$, und statt der im Falle $l=\infty$ errechneten Grenze (8) der Strombelastung ergibt sich jetzt bei endlicher Bahnlänge $l$ als Grenze zwischen schwacher und starker Injektion der Stromdichtewert

$$
\begin{aligned}
& 2 e(D / L) \operatorname{Cotg}(l / L) n_{\mathrm{A}^{-}} \\
\text {bzw. } & 2 e(D / l) n_{\mathrm{A}^{-}} .
\end{aligned}
$$

Der Gültigkeitsbereich, den das im folgenden entwickelte Lösungssystem abdeckt, ist aus Abb. $3 \mathrm{zu}$ ersehen. Wenn auch unsere Aufmerksamkeit im wesentlichen dem Bereich hoher Flußbelastungen gilt, besteht doch hinsichtlich der Größe der Injektion keine einschränkende Voraussetzung. Die Voraussetzung kurzer Bahngebiete bedeutet roh, daß wir uns etwa auf den Bereich $l \ll L$ beschränken ${ }^{10}$. Für diese kurzen Bahngebiete können wir die durch

10 Es wird sich allerdings zeigen, daß die zu entwickelnde Lösung auch Fälle mit größerer Bahnlänge $l$ noch ganz gut erfaßt und dies um so mehr, je höher die Strombelastung, je stärker die Injektion ist.

11 Wir haben in (24) und später auch in (28) die Gleich-
(12) regulierte Änderung des Defektelektronenstromes vernachlässigen und erhalten

$$
i_{\mathrm{p}}=-e D \frac{\mathrm{d} p}{\mathrm{~d} x}+e \mu p \mathbb{E}=\text { const }=i / 2
$$

bzw. für den Elektronenstrom

$$
i_{\mathrm{n}}=+e D \frac{\mathrm{d} n}{\mathrm{~d} x}+e \mu n E=\text { const }=i / 2 .
$$

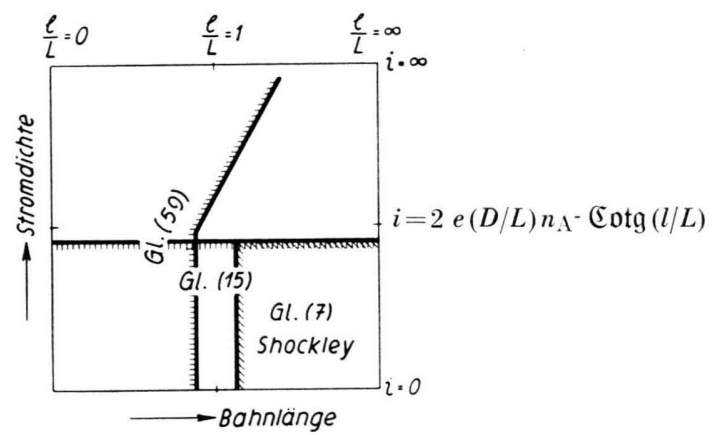

Abb. 3. Gültigkeitsbereich von verschiedenen p-n-Theorien.

Aus diesen Gleichungen können wir (E mit Hilfe von (11) eliminieren und erhalten z. B. für die Defektelektronenkonzentration im p-Gebiet aus (18) die Differentialgleichung

deren Lösung

$$
\frac{\mathrm{d} p}{\mathrm{~d} x}=\frac{i}{2 e D} \cdot \frac{n_{\mathrm{A}^{-}}}{2 p-n_{\mathrm{A}^{-}}},
$$

$$
p=n_{\mathrm{A}^{-}} \cdot\left\{\frac{1}{2}+\sqrt{\frac{i}{2 e D n_{\mathrm{A}^{-}}}(x+l)+\frac{1}{4}}\right\}
$$

man durch elementare Integration mit Berücksichtigung der Randbedingung (13) erhält. Führen wir mit Hilfe von (17) noch eine reduzierte Stromdichte

$$
\mathrm{i}=\frac{i}{2 e n_{\mathrm{A}}-D / l}
$$

ein, so wird schließlich:

$$
p=n_{\mathrm{A}^{-}} \cdot\left\{\frac{1}{2}+\sqrt{\mathrm{i}}([x / l]+1)+\frac{1}{4}\right\} .
$$

Den Verlauf der Elektronenkonzentration im p-Gebiet erhalten wir aus (23) und (5) ${ }^{11}$ :

$$
n=n_{\mathrm{A}^{-}} \cdot\left\{-\frac{1}{2}+\sqrt{\mathrm{i}([x / l]+1)+1 / 4}\right\}+n_{\mathrm{p}} .
$$

Schließlich liefert uns (11) mit (23), (22) und (8) den Ortsverlauf der Feldstärke

$$
\mathfrak{E}(x)=\mathfrak{V} / l \cdot \frac{\mathrm{i}}{\sqrt{\mathrm{i}([x / l]+1)+1 / 4}}
$$

gewichtskonzentration $n_{\mathrm{p}}=n_{\mathrm{i}}^{2} / n_{\mathrm{A}}{ }^{-}$der Elektronen im pGebiet, die wir in der Randbedingung (13) vernachlässigt haben, mit aufgeführt, um die Gleichungen formal für $x=-l$ in Ordnung zu halten. Das Glied $n_{\mathrm{p}}$ spielt jedoch sonst nur im Grenzfall $\mathfrak{i} \rightarrow 0$ eine Rolle (vgl. Anm. ${ }^{19}$ ). 
und den Potentialverlauf

$$
\begin{aligned}
V(x)-V\left(x_{\mathrm{p}}\right)=2 \mathfrak{B} & \left\{\sqrt{\mathbf{i}\left(\left[x_{\mathrm{p}} / l\right]+1\right)+\frac{1}{4}}\right. \\
& \left.-\sqrt{\mathbf{i}([x / l]+1)+\frac{1}{4}}\right\} .
\end{aligned}
$$

Durch die Gln. (23), (24) und (25) bzw. (26) wird das Geschehen in den Bahngebieten vollständig beschrieben. Dabei ist die reduzierte Stromdichte $i$ nach dem in der Einleitung und im Kleindruck bei Gln. (15), (16) und (17) Gesagten so festgelegt, $\mathrm{da} ß \mathrm{i} \ll 1$ den Gültigkeitsbereich der SноскLEYschen Theorie darstellt, während das Gebiet hoher Durchlaßströme mit $i \approx l$ beginnt.

Die Gln. (23) und (24) beschreiben im einzelnen die schon einleitend skizzierten Konzentrationsanhebungen, und zwar steigen bei hohen Stromdichten $i \gg 1$ beide Konzentrationen fast im ganzen Bahngebiet proportional mit der Wurzel aus der Stromdichte an, und zwar weit über die Dotierungskonzentration hinaus ${ }^{12}$. Abb. 4 zeigt den Verlauf der

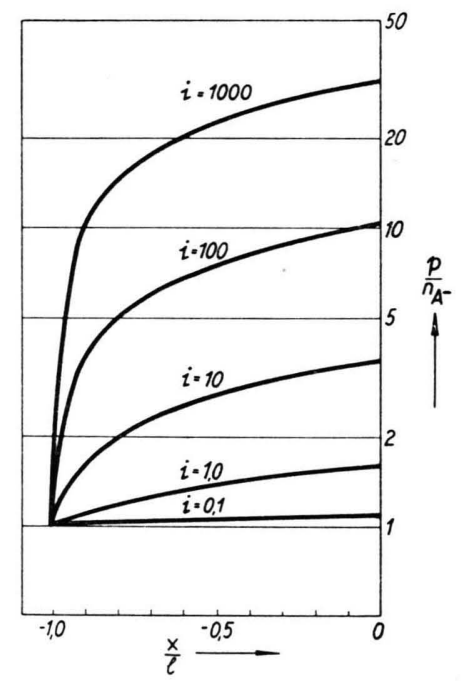

Abb. 4. Konzentrationsverlauf der Defektelektronen im p-Gebiet.

Defektelektronenkonzentration im p-Gebiet für verschiedene Werte von i. In Abb. 5 ist die Konzentration der Majoritätsträger und Minoritätsträger, der Defektelektronen und Elektronen am Ende der Raumladungszone bei $x=x_{\mathrm{p}}$ aufgetragen. Die Gln.

12 Die Konzentrationsanhebung kann natürlich nicht beliebig weit gehen. Ein Grund für ein allmähliches Abstoppen dieses Vorganges wird beispielsweise durch die bei sehr hohen Konzentrationen $p \rightarrow N_{\mathrm{V}}$ einsetzende Umladung der Akzeptoren (Übergang von Störstellenerschöpfung zu Störstellenreserve) und der damit verbundenen Abnahme von $n_{\mathrm{A}^{-}}$gegeben.
(23) und (24) liefern uns hierfür mit $x_{\mathrm{p}} \ll l$ (siehe Anm. ${ }^{13}$ ) die Beziehungen

$$
p\left(x_{\mathrm{p}}\right)=n_{\mathrm{A}^{-}} \cdot\left\{\frac{1}{2}+\sqrt{\mathbf{i}+\frac{1}{4}}\right\}
$$

und

$$
n\left(x_{\mathrm{p}}\right)=n_{\mathrm{A}^{-}} \cdot\left\{-\frac{1}{2}+\sqrt{\mathrm{i}+\frac{1}{4}}\right\}+n_{\mathrm{p}},
$$

die wir später als Randbedingungen bei der Behandlung der Raumladungszone benutzen werden. Man sieht daraus den Anstieg mit $\sqrt{i}$ für große Stromdichten.

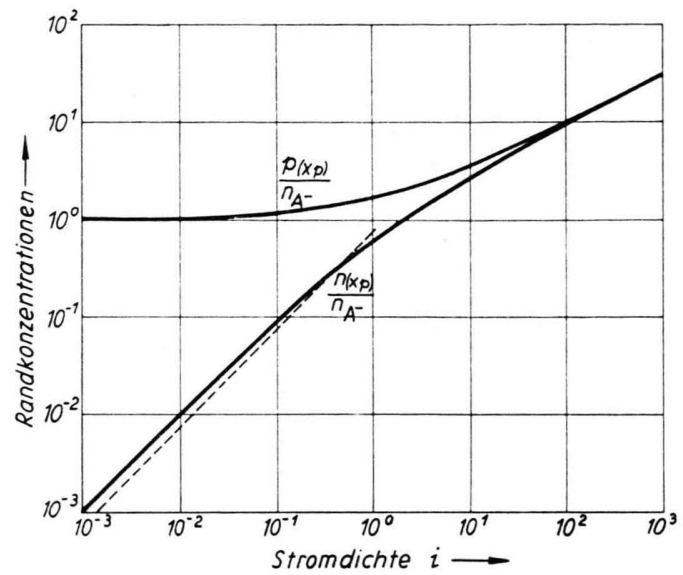

Abb. 5. Randkonzentrationen $p\left(x_{\mathrm{p}}\right)$ und $n\left(x_{\mathrm{p}}\right)$ an der Grenze zwischen Raumladungsgebiet und p-leitendem Bahngebiet in Abhängigkeit von der Stromdichte. .... . Randkonzentration $n\left(x_{\mathrm{p}}\right)$ nach Gl. (15) mit $l=L$.

Für kleine Stromdichten folgt aus (28) :

$$
n\left(x_{\mathrm{p}}\right)=n_{\Lambda^{-}} \cdot \mathrm{i} \text {. }
$$

Vergleichen wir dies mit der aus (15) und (22) folgenden Beziehung

$$
n_{\mathrm{p}} e^{U / \mathfrak{B}}=n\left(x_{\mathrm{p}}\right)=n_{\mathrm{A}^{-}} \cdot \mathrm{i} \cdot(L / l) \mathfrak{T} \mathfrak{g}(l / L),
$$

die zwar $i \ll 1$ voraussetzt, aber in bezug auf $l / L$ keinerlei Einschränkungen unterliegt, so sehen wir, $\mathrm{da} \beta$ abgesehen von der selbstverständlich zu fordernden Übereinstimmung für $l / L \rightarrow 0$ auch die bei größeren $l / L$-Werten eintretenden Unterschiede nicht allzu kraß sind. (Siehe z. B. in Abb. 5 die gestrichelt eingetragene Kurve für $l / L=1$.) Die hier vorgetragene und von der Voraussetzung kurzer Bahngebiete ausgehende Theorie erfaßt also auch noch für $l=L$

${ }^{13}$ Die Breite der Raumladungszone (quantitative Werte siehe Abschnitt II) bleibt stets in der Größenordnung unter $1 \mu$, kann also im Vergleich zu den technisch realisierbaren Bahnlängen von einigen $100 \mu$ bedenkenlos vernachlässigt werden, 
die Verhältnisse ganz gut. Zur weiteren Prüfung wird in Abb. 6 die Änderung des Defektelektronenstromes im p-Gebiet gezeigt. Sie geht in „kurzen“ Bahngebieten mit $(l / L)^{2}$ nach Null, und auf diesem Verschwinden beruht ja die Ausgangsgleichung (18) der vorliegenden Theorie. Aber auch innerhalb von endlichen Bahngebieten (z. B. $l=L$ ) ändert sich der Defektelektronenstrom namentlich bei stärkeren Injektionen $i \gg 1$ nicht sehr stark. Dadurch ist die vorgetragene Theorie auch für solche Fälle mit guter Annäherung gerechtfertigt und erfaßt somit die technisch wichtigen Anordnungen, bei denen die Bahnlänge $l$ kaum wesentlich größer als die Diffusionslänge $L$ zu sein pflegt.

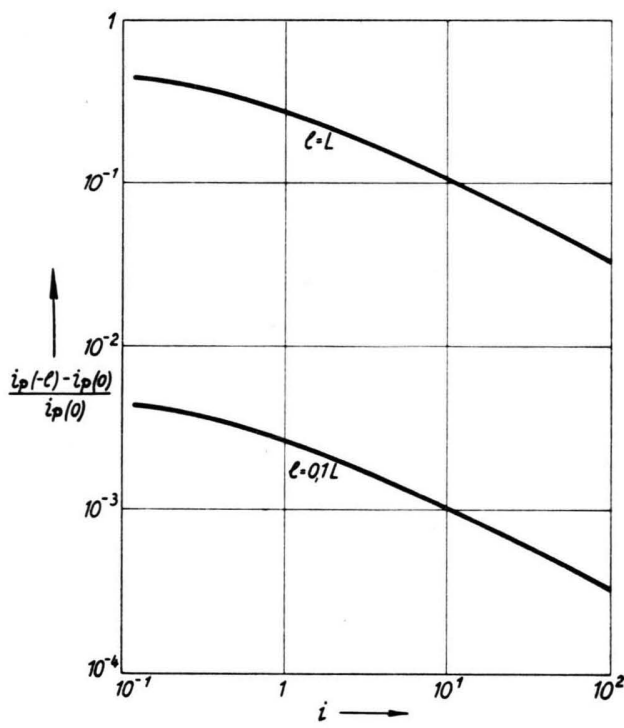

Abb. 6. Relative Änderung des Defektelektronenstromes im Bahngebiet.

Wir wollen hier die Berechnung der Kurve in Abb. 6 nur skizzieren. Die Änderung des Defektelektronenstromes $i_{\mathrm{p}}(-l)-i_{\mathrm{p}}(0)$ im Bahngebiet erhält man durch Integration von (12). Den Rekombinationsüberschuß ${ }^{14}$

$$
\mathfrak{R}=\frac{n p-n_{\mathrm{i}}^{2}}{T_{1} n_{\mathrm{i}}+T_{2} n+T_{3} p},
$$

kann man mit (23) und (24) als Funktion des Ortes darstellen. Die Zeitkonstanten $T_{2}$ und $T_{3}$ in (29) sind im wesentlichen mit den Lebensdauern $\tau_{\mathrm{p}}$ und $\tau_{\mathrm{n}}$ im n- und p-Gebiet identisch und können in unserem Fall vollständiger Symmetrie durch die Diffusionslänge $L$ ausgedrückt werden

$$
T_{2}=T_{3}=L^{2} / D
$$

14 Vgl. hierzu Anm. ${ }^{5}$, S. 100.
Nimmt man noch $T_{1}$ in der Größenordnung von $T_{2}$ bzw. $T_{3}$ an, so lassen sich in (29) die Glieder mit $n_{\mathrm{i}}$ vernach. lässigen; man kann dann (12) gezchlossen integrieren und erhält

$\frac{i_{\mathrm{p}}(-l)-i_{\mathrm{p}}(0)}{i_{\mathrm{p}}(0)}=\frac{1}{3 \mathrm{i}^{2}} \cdot \frac{l^{2}}{L^{2}} \cdot\left\{\frac{1}{4}+\gamma \overline{\mathrm{i}+\frac{1}{4}}\left(\mathrm{i}-\frac{1}{2}\right)\right\}$.

Schließlich ist hier noch eine Bemerkung zu machen, die an Gl. (25) anschließt, nach der ,fast“ im ganzen Bahngebiet die Feldstärke mit der Wurzel aus der Stromdichte ansteigt. Insbesondere für den Randwert bei $x=x_{\mathrm{p}}$ erhält man wegen $\left|x_{\mathrm{p}}\right| \ll l$ :

$$
E\left(x_{\mathrm{p}}\right)=\frac{\mathfrak{V}}{l} \cdot \frac{\mathrm{i}}{\sqrt{\mathrm{i}+1 / 4}} \text {. }
$$

Im Bereich unmittelbar vor der Metallkontaktierung bei $x=-l$ muß dem steilen Abfall der Konzentrationen (Abb. 4) nach (11) ein starkes örtliches Anwachsen der Feldstärke entsprechen. Man hat dort daher bei hohen Stromdichten einen erheblichen Gradienten der Feldstärke, dem nach der Poissonschen Gleichung auch nennenswerte Raumladungen entsprechen. Die nach (5) geforderte Quasineutralität bleibt daher bei hohen Stromdichten $\mathrm{i} \gtrsim l /\left(\sqrt{ } 8 \cdot x_{\mathrm{op}}\right) \gtrsim 1000$ (siehe Anm. $\left.{ }^{15}\right)$ unmittelbar vor der Grenze des Bahngebietes bei $x=-l$ nicht mehr hinlänglich gewahrt, so daß unsere Näherung dann von dort her zu versagen beginnt.

Das hier geschilderte Geschehen in den Bahngebieten ist im übrigen von den speziellen Annahmen (kurze Bahngebiete, sperrfreie Kontakte) in wesentlichen Zügen unabhängig. Es lassen sich beispielsweise auch für unendlich ausgedehnte Bahngebiete mit einigem Aufwand brauchbare Näherungslösungen angeben; auch sie zeigen ein Anwachsen der Konzentrationen mit der Wurzel aus der Stromdichte. Anschaulich kann man dieses Anwachsen der Konzentrationen vielleicht folgendermaßen verstehen:

In dem schmalen Übergangsgebiet zwischen den neutralen p- und n-Bahngebieten "springen" die Konzentrationen gleichsam um den Betrag der Dotierungskonzentrationen (Abb. 7). Vergleicht man symmetrisch zum p-n-Übergang gelegene Punkte miteinander, so ist links die Defektelektronenkonzentration höher als rechts. Die Feldstärke ist aber an beiden Punkten gleich. Der Feldanteil des Defektelektronenstromes ist daher links größer als rechts. Der Defektelektronenstrom soll jedoch auf beiden

15 Die Debye-Länge $x_{0 p}=\sqrt{\varepsilon \mathfrak{V} / 4 \pi e n_{\Lambda^{-}}}$liegt in der GröBenordnung $0,1 \mu$, die Bahnlänge in der Größenordnung 100 bis $1000 \mu$. 
Seiten gleich sein, und zwar gleich $i / 2$. Den Ausgleich schafft der Diffusionsanteil des Defektelektronenstromes, der links dem zu großen Feldanteil entgegenwirkt und ihn auf $i / 2$ reduziert und der rechts den zu kleinen Feldanteil unterstützt und ihn auf $i / 2$ erhöht. Mit wachsender Stromdichte und damit wachsender Feldstärke müssen auch diese ausgleichenden Diffusionsströme anwachsen und deshalb die Konzentrationsgradienten und damit auch die Konzentrationen größer werden.

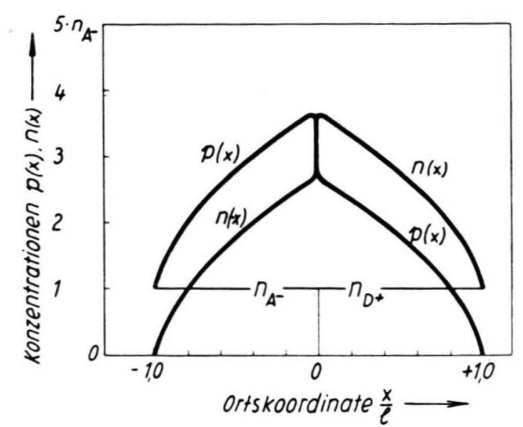

Abb. 7. Konzentrationsverlauf der Defektelektronen und Elektronen bei hoher Strombelastung in einem symmetrischen p-n-Gleichrichter $(i=10)$.

\section{Die Raumladungszone}

Der mathematischen Behandlung des Raumladungsgebietes müssen einige grundsätzliche Bemerkungen vorausgeschickt werden. Die Aufteilung in quasineutrale Bahngebiete und in eine nicht neutrale Raumladungszone ist ja eine Willkür. In Wirklichkeit gehen beide Zonen bzw. Gebiete ineinander über bzw. es kann gar nicht streng zwischen ihnen unterschieden werden.

Eine einheitliche Behandlung beider Gebiete scheitert aber an mathematischen Schwierigkeiten. So muß man in den Bahngebieten die Näherungsannahme strikter Neutralität machen, die in der Raumladungszone natürlich kraß durchbrochen wird, und bei der folgenden Behandlung der Raumladungszone werden wir die Voraussetzung $V(x)-V(0) \ll \mathfrak{B}$ machen müssen, die wieder in den Bahngebieten nicht erfüllt ist.

Es hat sich nun gezeigt, daß der Versuch, die beiden so verschieden behandelten Zonen mit streng erfüllten Randbedingungen aneinander anzuschließen, nicht zu einer mathematischen Behandlung führt, die den physikalischen Sachverhalt vernünftig wieder- gibt. Wir müssen vielmehr von einer Tatsache Gebrauch machen, die die ganze Unterscheidung zwischen den neutralen Bahnzonen und einer Raumladungszone physikalisch so zwingend nahelegt. Es ist dies die krasse Verschiedenheit der Längenmaßstäbe, die sich bei der Behandlung der Bahnzonen und bei der Behandlung der Raumladungszone als charakteristisch herausstellen.

Bei den „kurzen“ Bahngebieten ist die charakteristische Länge die Länge $l$ eines solchen Bahngebietes selbst. Sie wird zunächst zwar klein gegen die Diffusionslänge $L$ vorausgesetzt, ihre Größe beträgt aber in der Praxis immer noch mindestens $100 \mu$. Damit ist sie sehr groß gegenüber allen Werten der Deвуe-Länge $x_{0}$, die selbst in eigenleitendem Ge zum Beispiel nur $1 \mu$ beträgt, aber bei größeren Trägerdichten noch viel kleinere Werte hat.

Es wird sich nun im folgenden zeigen, daß die Raumladungseffekte in Abständen von wenigen Deвуе-Längen von der Dotierungsgrenze bereits weitgehend abgeklungen sind, so daß die eigentliche Raumladungszone zwischen $x_{\mathrm{p}}$ und $x_{\mathrm{n}}$ nur einige Debye-Längen umfaßt und deshalb als schmal gegenüber der Bahnlänge $l$ angesehen werden darf. Andererseits haben sich in der Raumladungszone bereits nach wenigen Debye-Längen mit großer Näherung dieselben Verhältnisse eingestellt, wie sie bei $x= \pm \infty$ eintreten würden, falls die Gesetze der Raumladungszone nach rechts und links beliebig weit gelten würden. Verständlicherweise ist es mathematisch einfacher, mit diesen Grenzwerten für $\pm \infty$ zu rechnen.

Wir werden daher auf die exakte Festlegung einer Grenze $x_{\mathrm{p}}$ der Raumladungszone verzichten und statt dessen diese Zone durch die Forderung

$$
x_{0} \ll\left|x_{\mathrm{p}}\right| \ll l
$$

nur größenordnungsmäßig einengen. Auf Grund der linken Seite von (33) können wir bei einer Behandlung der Raumladungszone, in der die charakteristische Länge $x_{0}$ ist, $x_{\mathrm{p}}=-\infty$ setzen. Für das Bahngebiet ist jedoch auf Grund der rechten Seite von (33) $x_{\mathrm{p}}=0$ zu setzen, und deshalb wurden die Anschlußgln. (27), (28) und (32) auf Grund dieser Vereinfachung gewonnen. Die Ungleichung (33) zeigt also, daß der Anschluß zwischen Raumladungszone und Bahngebiet sinngemäß vorgenommen wird, obwohl die Potentialtangente im Raumladungsgebiet für $x \rightarrow \infty$ mit der Potentialtangente im Bahngebiet 
für $x \rightarrow 0$ zur Deckung gebracht werden wird [Festlegung des $\left(p(0) / n_{\mathrm{A}^{-}}\right)$-Wertes gemäß (46) ].

Für die Behandlung der Raumladungszone können wir wieder, und zwar mit noch größerer Berechtigung als in den vergleichsweise großen Bahngebieten, die Gln. (18) und (19) zugrunde legen. Dagegen tritt jetzt an Stelle der in I benutzten Quasineutralitätsbedingung (5) die Porssonsche Gleichung, die im p-dotierten Bereich $x \leqq 0$ links der Dotie- rungsgrenze, auf den wir uns hier beschränken wollen,

$$
\frac{\mathrm{d}^{2} V}{\mathrm{~d} x^{2}}=-\frac{4 \pi Q}{\varepsilon}=-\frac{4 \pi e}{\varepsilon}\left(p-n-n_{\mathrm{A}^{-}}\right)
$$

lautet.

Damit schließen wir die prinzipiellen Vorbemerkungen $a b$ und beginnen mit einer Integration der Gln. (18) und (19) :

$$
\begin{aligned}
& p(x)=\exp [-(V(x)-V(0)) / \mathfrak{B}]\left\{p(0)-\frac{i}{2 e D} \int_{\xi=0}^{\xi=x} \exp [+(V(\xi)-V(0)) / \mathfrak{B}] \mathrm{d} \xi\right\}, \\
& n(x)=\exp [+(V(x)-V(0)) / \mathfrak{B}]\left\{n(0)+\frac{i}{2 e D} \int_{\xi=0}^{\xi=x} \exp [-(V(\xi)-V(0)) / \mathfrak{B}] \mathrm{d} \xi\right\} .
\end{aligned}
$$

Man kann nun (35) und (36) für den Fall der hohen Durchlaßbelastung sehr vereinfachen. Wie bereits einleitend bemerkt wurde, werden wir ja erwarten, daß bei hohen Flußspannungen die Diffusionsspannung $V_{\mathrm{D}}$ weitgehend abgebaut und der Potentialverlauf in der Raumladungszone nivelliert wird. Wir wollen daher annehmen, daß - wenigstens in einem gewissen Strombereich - die Änderungen des Potentials in der Raumladungszone hinreichend klein sind:

$$
\frac{V(x)-V(0)}{\mathfrak{B}}=\varphi \ll 1 \quad \text { in } x_{\mathrm{p}} \leqq x<x_{\mathrm{n}} .
$$

Damit können wir die Exponentialfunktion in (35) und (36) durch einen linearen Ansatz

$$
e^{\varphi}=1+\varphi \text { bzw. } e^{-\tau}=1-\varphi
$$

annähern und erhalten unter Vernachlässigung der in $\varphi$ quadratischen Glieder

$$
\begin{gathered}
\pm \frac{i}{2 e D} \varphi(x) \int_{0}^{x} \varphi(\xi) \mathrm{d} \xi: \\
p(x)=p(0)-\frac{i}{2 e D} x-p(0) \varphi(x) \\
+\frac{i}{2 e D} x \varphi(x)-\frac{i}{2 e D} \int_{0}^{x} \varphi(\xi) \mathrm{d} \xi, \\
n(x)=n(0)+\frac{i}{2 e D} x+n(0) \varphi(x) \\
+\frac{i}{2 e D} x \varphi(x)-\frac{i}{2 e D} \int_{0}^{x} \varphi(\xi) \mathrm{d} \xi .
\end{gathered}
$$

Da ferner auf Grund unserer Symmetrieannahmen die Elektronen- und die Defektelektronenkonzentra- tion an der Dotierungsgrenze gleich sein müssen

$$
p(0)=n(0),
$$

ergibt sich durch Differenzbildung von (39) und (40) die einfache Beziehung

$$
p(x)-n(x)=-2 p(0) \varphi-\frac{i}{e D} x
$$

Gehen wir mit dieser Gl. (42) in die Porssonsche Gl. (34) ein und benutzen wir die durch (22) eingeführte reduzierte Stromdichte $i$, so erhalten wir für den Potentialverlauf die Differentialgleichung:

$$
\frac{\mathrm{d}^{2} \varphi}{\mathrm{d} x^{2}}=\frac{4 \pi e n_{\mathrm{A}^{-}}}{\varepsilon \mathfrak{B}} \cdot\left\{\frac{2 p(0)}{n_{\mathrm{A}^{-}}} \varphi+2 \mathrm{i}^{\frac{x}{l}}+1\right\} .
$$

Man bestätigt durch Ausdifferenzieren, daß

$$
\varphi=\frac{n_{A^{-}}}{2 p(0)}\left\{e^{x / x_{0}}-1-2 \mathrm{i} \frac{x}{l}\right\}
$$

mit einer Debye-Länge ${ }^{16}$

$$
x_{0}=\sqrt{\varepsilon \mathfrak{B} / 4 \pi e 2 p(0)}
$$

ein Integral von (43) ist. Diese Lösung (44) ist in Abb. 8 dargestellt, wobei sie antisymmetrisch in das Gebiet $x>0$ ergänzt wurde. Der Kurvenverlauf - links ein linearer Potentialverlauf, darauf folgend eine durch Raumladungen bedingte Potentialstufe und dann rechts wieder ein linearer Potentialabfall - entspricht in seinem ganzen Charakter dem, was aus physikalischen Gründen für den betrachteten Übergang zwischen den beiden Bahngebieten $\mathrm{zu}$ fordern ist. Legen wir schließlich den in (44)

16 Zum Begriff der Debye-Länge vergleiche man Anm. ${ }^{3}$, S. 101, Fußnote 1. 
bislang noch unbestimmten Konzentrationswert $p(0)$ folgendermaßen fest:

$$
p(0)=\mathrm{n}_{\mathrm{A}^{-}} \cdot \sqrt{\mathrm{i}+\frac{1}{4}},
$$

so erhalten wir

$\varphi(x) \approx \frac{1}{2 \sqrt{i}+\frac{1}{4}}\left\{e^{x / x_{0}}-1-2 \mathrm{i}^{x}\right\}$ für $x<0$,

bzw. mit Hilfe von (32)

$\varphi(x)=\frac{1}{2 \sqrt{\mathfrak{i}+\frac{1}{4}}}\left(e^{x / x_{0}}-1\right)-\stackrel{\mathfrak{E}\left(x_{\mathrm{p}}\right) x}{\mathfrak{B}}$ für $x<0$.

Man sieht, daß der für $x \rightarrow-\infty$ allein übrigbleibende lineare Potentialabfall dem richtigen Bahnfeldstärkenwert (E $\left(x_{\mathrm{p}}\right)$ entspricht, womit die Festsetzung (46) gerechtfertigt ist.

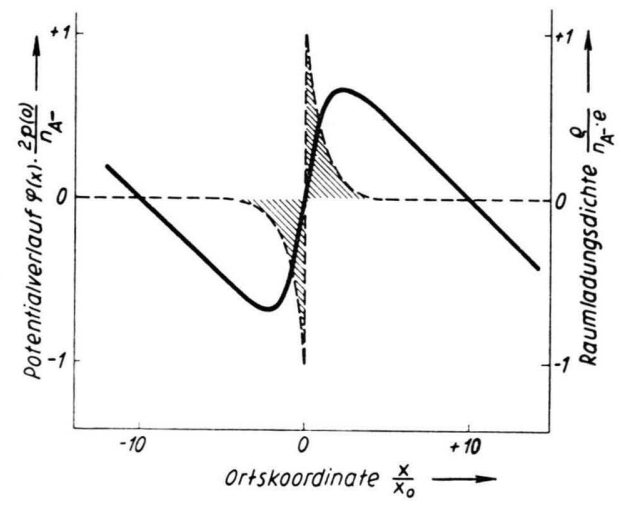

Abb. 8. Potentialverlauf und Raumladungsdichte in der Nähe der Dotierungsgrenze. $\longrightarrow$ Potentialverlauf: $V(x)-V(0)$ in Einheiten für $2 \dot{i}\left(x_{0} / l\right)=0,1 ; \ldots$. Raumladungsdichte $\varrho(x)$ in Einheiten $e n_{\Lambda^{-}}$.

Die Verwendung von (47) in (39) und (40) liefert schließlich ${ }^{17}$

$p(x)=n_{A^{-}} \cdot\left\{-\frac{1}{2}\left(e^{x / x_{0}}-1\right)+\sqrt{i+\frac{1}{4}}+\ldots\right\}$,

$n(x)=n_{\Lambda^{-}} \cdot\left\{+\frac{1}{2}\left(e^{x / x_{0}}-1\right)+1 / i+\frac{1}{4}+\ldots\right\}$.

Wie (48) liefern auch (49) und (50) für $x \rightarrow-\infty$ die richtigen ${ }^{18}$, in diesem Fall durch (27) und (28) angegebenen Randwerte des Bahngebietes.

17 In den Gln. (39) und (40) ergibt sich anscheinend eine eindeutige Aufteilung in Hauptglieder, nämlich die beiden ersten $\varphi(x)$ nicht enthaltenden Summanden, und in Korrekturglieder erster Ordnung, nämlich die drei letzten Summanden. Diese Klassifizierung der Summanden der Gln. (39) und (40) erweist sich jedoch auf Grund der für das Raumladungsgebiet entwickelten Lösung (47) als nicht haltbar. Aus (47) und (46) folgt nämlich, daß in dem durch (37) begrenzten Gültigkeitsbereich dieser Lösung stets

$$
(i / 2 \text { e } D) x=\mathrm{i} \cdot n_{\Lambda^{-}}(x / l) \ll p(0)
$$

bleiben muß. Der 2. Summand in (39) stellt somit im Vergleich zu $p(0)$ schon ein Korrekturglied dar. Damit werden aber die beiden letzten Summanden in (39), die diesen 2 . Term explizit bzw. implizit neben $\varphi$ als Faktor ent-
Die Abnahme der Debye-Länge

$$
x_{0}=\sqrt{\frac{\varepsilon \mathfrak{B}}{4 \pi e n_{1}-1^{-}}} \cdot \frac{1}{\sqrt{2}\left(\mathfrak{i}+\frac{1}{4}\right)^{3 / 4}}
$$

mit wachsender Stromdichte $i$ beschreibt das räumliche Schrumpfen der Raumladungszone, während das allmähliche Verschwinden der von der Raumladung aufgebauten Spannung durch den Faktor $1 / 2 \sqrt{i}+\frac{1}{4}$ vor dem ersten Term in (48) bestimmt wird. Dies alles wird auch in den Abb. 9 und 10 sichtbar, in denen die Gesamtlösung nach (47), (49) und (50) dargestellt ist. Im übrigen liefern die Gln.

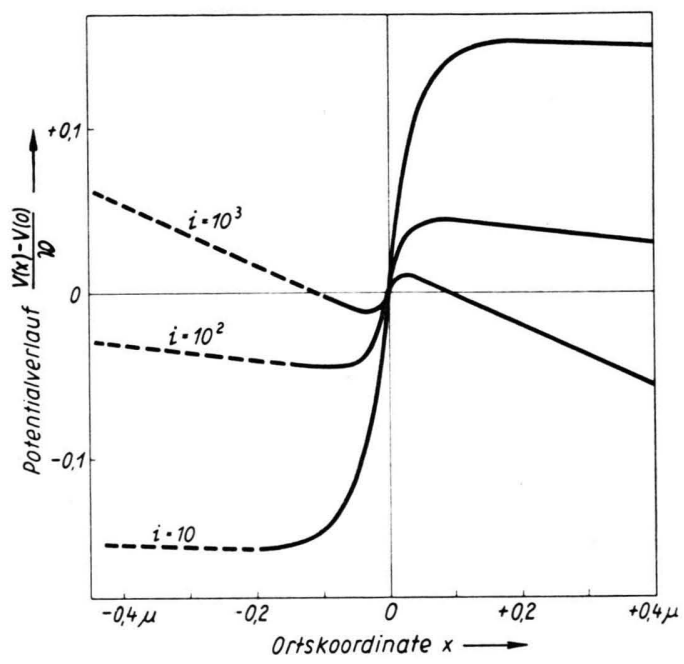

Abb. 9. Potentialverlauf in der Raumladungszone. $x_{\mathrm{op}}=0,1 \mu ; l=200 \mu ; \cdots$. Verlauf nach Gl. (26).

(47) bzw. (48) zusammen mit (37) für den Potentialwert einer außerhalb des eigentlichen Raumladungsgebietes liegenden Stelle $x_{\mathrm{p}}\left(\left|x_{\mathrm{p}}\right| \gg x_{0}\right)$ den Ausdruck

$$
V\left(x_{\mathrm{p}}\right)-V(0)=-\mathfrak{B} \frac{1}{2 \sqrt{\mathrm{i}+\frac{1}{4}}}-\mathfrak{V} \frac{\mathrm{i}}{\sqrt{\mathrm{i}+\frac{1}{4}}} \cdot \frac{x_{\mathrm{p}}}{\mathfrak{W}},
$$

den wir in Teil III benutzen wollen.

halten, zu Korrekturgliedern zweiter Ordnung entwertet. Sie dürfen daher bei konsequenter Benutzung des linearen Näherungsansatzes (38) gar nicht berücksichtigt werden. Die gleiche Überlegung gilt auch für Gl. (40). Für die Ableitung der Lösung (47) sind die Betrachtungen ohne Bedeutung, da bei der Gewinnung der entscheidenden Gl. (42) die beiden letzten Glieder in (39) und (40) sich ohnehin exakt wegheben. Bei der Berechnung der Konzentrationsverteilungen (49) und (50) sind dagegen aus den genannten Gründen nur die 3 ersten Summanden in (39) und (40) zu berücksichtigen.

18 Der Term $n_{\mathrm{p}}$ in $(28)$ ist wegen $n_{\mathrm{p}} \ll n_{\mathrm{A}^{-}}$schon bei $\mathrm{i} \approx 1$ bedeutungslos. Die im Abschnitt II gegebene Behandlung des Raumladungsgebietes beschränkt sich aber von vornherein auf starke Strombelastungen. 
Durch das Schrumpfen der Raumladungszone und das Anwachsen der Bahnfeldstärke wird auch die Feldstärke an der Dotierungsgrenze

$$
\mathfrak{E}(0)=-\frac{\mathfrak{B}}{x_{0}} \frac{1}{2 \sqrt{\mathrm{i}+\frac{1}{4}}}+\frac{\mathfrak{B}}{l} \frac{\mathrm{i}}{\sqrt[V]{ } \mathrm{i}+\frac{1}{4}}
$$

allmählich abgebaut (Abb.9) und schließlich für i $>l / 2 x_{0}$ sogar in die Richtung der Bahnfeldstärke

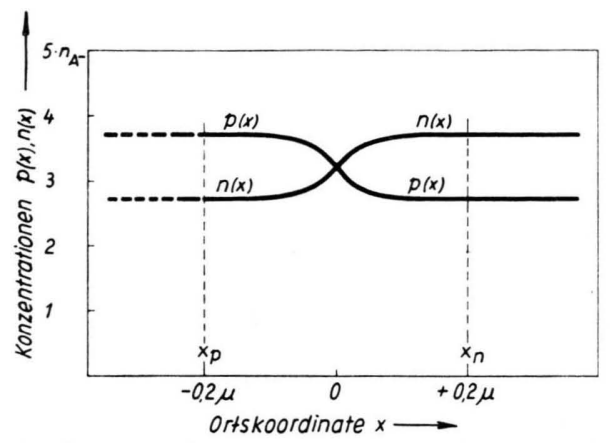

Abb. 10. Konzentrationsverteilungen in der Raumladungszone. $x_{\mathrm{op}}=0,1 \mu ; i=10$. Verlauf nach den Gln. (23) und (24).

gekehrt. Sie bleibt jedoch stets kleiner als die Bahnfeldstärke, deren Wert sie erst im Grenzfall $i \rightarrow \infty$ erreicht. Praktisch dürfte jedoch die zur Umkehrung der Feldrichtung erforderliche Stromdichte schon kaum mehr erreicht werden.

\section{Die Strom-Spannungs-Kennlinie}

Wir haben in Teil I den Potentialverlauf im Bahngebiet berechnet und in Teil II den Potentialverlauf in der Raumladungszone, wobei wir die Stromdichte $i$ jeweils als Parameter benutzt haben (Abb. 11). Wir sind damit jetzt in der Lage, den zu $i$ zugehörigen Wert der äußeren Spannung $U$ anzugeben. Berücksichtigen wir, daß im stromlosen Zustand, also bei $U=0$, zwischen p-Gebiet und n-Gebiet die Potentialdifferenz ${ }^{19}$

$$
-V_{\mathrm{D}}=-\mathfrak{Z} \ln \left(\frac{n_{\mathrm{A}^{-}} n_{\mathrm{D}^{+}}}{n_{\mathrm{i}^{2}}}\right)=-\mathfrak{V} \ln \left(\begin{array}{c}
n_{\mathrm{A}^{-}} \\
n_{\mathrm{i}}
\end{array}\right)^{2}
$$

besteht, so erhalten wir

$$
\begin{aligned}
U & =V(-l)-V(+l)+V_{\mathrm{D}} \\
& =2\{V(-l)-V(0)\}+V_{\mathrm{D}} .
\end{aligned}
$$

Die Potentialdifferenz

$$
V(-l)-V(0)=V(-l)-V\left(x_{\mathrm{p}}\right)+V\left(x_{\mathrm{p}}\right)-V(0)
$$

19 Man vgl. Anm. ${ }^{3}$, S. 92. können wir nun mit (26) und (52) angeben. Beachten wir dabei, daß wir, um beide Lösungen aneinander anzuschließen, $\left|x_{\mathrm{p}}\right| \ll l$ setzen müssen und können [man vgl. Gl. (33)], so hebt sich der 2. Summand in (52) gegenüber dem Glied i $x_{\mathrm{p}} / l$ unter der Wurzel in (26) gerade auf und wir erhalten aus (55)

$$
U=4 \mathfrak{V}\left\{\sqrt{i}+\frac{1}{4}-\frac{1}{2}\right\}+\left\{V_{D}-\mathfrak{Z} \frac{1}{V i+\frac{1}{4}}\right\} .
$$

Dabei ist nach dem vorher Gesagten der von der Bahnfeldstärke herrührende 2. Term in (52) jetzt in den 1. Summanden von (57) - in den Spannungsabfall über den Bahngebieten also - mit einbezogen; der in diesem Sinne korrigierte Spannungs-

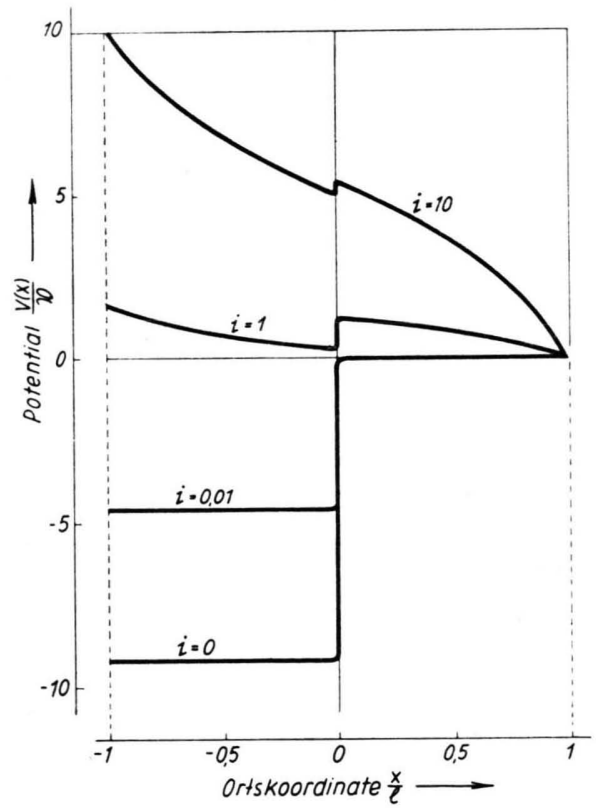

Abb. 11. Potentialverlauf im p-n-Gleichrichter bei niedrigen und hohen Injektionen.

abfall über der Raumladungszone, der 2. Summand in (57), entspricht in seinem Verhalten unseren einleitend ausgesprochenen Erwartungen: Er bleibt stets kleiner als der Wert $V_{\mathrm{D}}$, dem er sich mit wachsender Stromdichte asymptotisch nähert. Für $\mathrm{i} \gg 1$ können wir diesen Term praktisch gleich $V_{\mathrm{D}}$ setzen und erhalten dann aus (57) mit unserer Definitionsgleichung (22) die Strom - Spannungs-Kennlinie $i=\frac{2 e D n_{\mathrm{A}^{-}}}{l}\left(\frac{U-V_{\mathrm{D}}}{4 \mathfrak{B}}\right)^{2}=\frac{e \mu n_{\mathrm{A}^{-}}}{2 l} \cdot \frac{1}{4 \mathfrak{B}} \cdot\left(U-V_{\mathrm{D}}\right)^{2},(58)$ d. h. also einen quadratischen Anstieg der Stromdichte mit der Spannung $U-V_{\mathrm{D}}$, die über den Bahngebieten abfällt. 
Wir haben damit das Ziel unserer Betrachtungen, das Verhalten des p-n-Gleichrichters bei hohen Durchlaßströmen zu ermitteln, erreicht. Wir möchten jedoch nicht schließen, ohne einen Schönheitsfehler zu beseitigen, den Gl. (57) noch enthält.

Die in Abschnitt I vorgetragene Behandlung der Bahngebiete und damit der 1. Summand in (57) gelten sowohl für niedrige als auch für hohe Injektionen, also für $\dot{i}=0$ bis $\dot{i}=\infty$; dagegen ist die in Abschnitt II durchgeführte Behandlung des Raumladungsgebietes an die Näherungsannahme (37) und damit an die Voraussetzung $\mathrm{i} \gg 1$ gebunden. Der 2. Summand in (57) gilt daher ebenfalls nur für hohe Durchlaßströme. Dieser Mangel läßt sich nun dadurch beheben, daß wir im Endergebnis, also in der Gl. (57), die lineare Vereinfachung (38) durch eine formale Umformung wieder aufheben. Schreiben wir nämlich (57) in der Form

$$
\begin{array}{rl}
U=4 & \mathfrak{Z}\left\{\sqrt{\hat{\mathfrak{i}}+\frac{1}{4}}-\frac{1}{2}\right\} \\
+\left\{V_{\mathrm{D}}-\mathfrak{V} \ln \left[\frac{\frac{1}{2}+\sqrt{\mathrm{i}+\frac{1}{4}}}{-\frac{1}{2}+V / \mathfrak{i}+\frac{1}{4}+n_{\mathrm{i}}{ }^{2} / n_{\mathrm{A}^{-2}}}\right]\right\},
\end{array}
$$

so beschreibt diese Beziehung jetzt den ganzen Strombereich ${ }^{20} 0 \leqq \mathrm{i}<\infty$ richtig, wie man folgendermaßen einsieht:

Im Bereich $i \gg 1$ geht das Argument des Logarithmus in (59) gegen 1; wir können dann den Logarithmus nach Gliedern $1 / 2 \sqrt{\mathfrak{i}+\frac{1}{4}}$ entwickeln und erhalten wieder (57). Im Bereich $i \ll 1$ kann man die Glieder $\sqrt{\mathfrak{i}+\frac{1}{4}}$ im Logarithmus nach $i$ entwickeln und erhält die Beziehung

$$
U=V_{\mathrm{D}}+\mathfrak{V} \ln \left(\mathrm{i}+n_{\mathrm{i}}{ }^{2} / n_{\mathrm{A}^{-}}{ }^{2}\right),
$$

die mit (54) und (22) in die Kennliniengleichung (16) bei niedrigen Injektionen übergeht ${ }^{21}$.

Die Gl. (59) beschreibt also die Bereiche $\mathrm{i} \ll 1$ und $i \gg 1$ richtig, und wir können daher hoffen, daß sie auch im Übergangsgebiet $i \approx 1$ stimmt. Das bestätigt sich auch, wenn wir uns klarmachen, daß wir mit (27) und (28) den logarithmischen Term in (59) auch in der Form

$$
\ln \frac{p\left(x_{\mathrm{p}}\right)}{n\left(n_{\mathrm{p}}\right)} \text { bzw. } \ln \frac{p\left(x_{\mathrm{p}}\right)}{p\left(x_{\mathrm{n}}\right)} \text { bzw. } \ln \frac{n\left(x_{\mathrm{n}}\right)}{n\left(x_{\mathrm{p}}\right)}
$$

schreiben können. Der in $\mathfrak{Z}$ gemessene Potentialabfall über der Raumladungszone muß sich aber nun stets durch den Ausdruck (61) darstellen lassen,

20 Um auch das Nullgebiet der Strom-Spannungs-Kennlinie mit zu erfassen, ist im Nenner des logarithmischen Gliedes in (57), der nur im Grenzfall $i \rightarrow 0$ merkliche Summand $n_{\mathrm{i}}^{2} / n_{\mathrm{A}^{-2}}$ eingefügt. Man vgl. hierzu auch Gl. (61) und Anm. ${ }^{11}$. wenn in der Raumladungszone praktisch BoLtzmannGleichgewicht herrscht, wenn also dort Feld- und Diffusionsströme groß gegenüber dem resultierenden Gesamtstrom sind, sich also gegenseitig nahezu vollständig wegkompensieren müssen. Wie man nun aus Abb. 9 entnimmt, ist z. B. bei $\mathfrak{i}=10$ die Feldstärke im Raumladungsgebiet dem Betrage nach außerordentlich groß, verglichen mit der Feldstärke in den Bahngebieten, während nach Abb. 10 die Konzentrationen im Raumladungsgebiet und in den

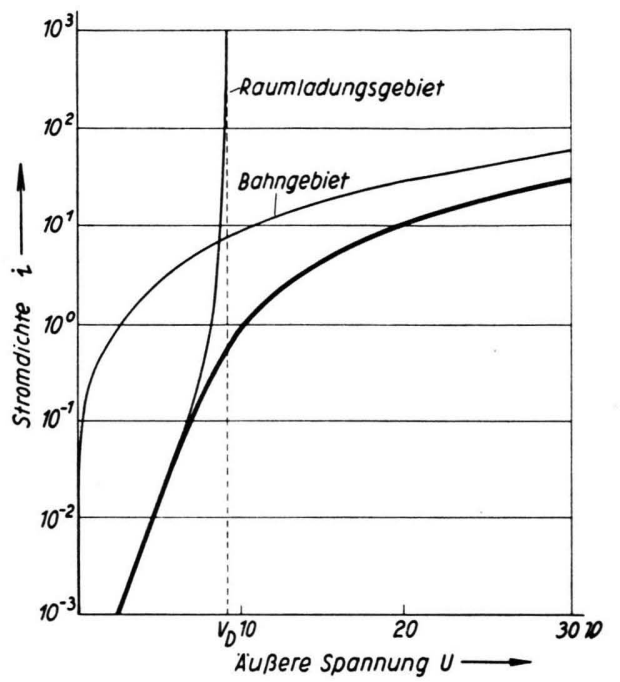

Abb. 12. Strom-Spannungs-Kennlinie eines p-n-Gleichrichters. Aufteilung der äußeren Spannung $U$ auf Raumladungsgebiet und Bahngebiete.

Bahngebieten etwa gleich groß sind. Bei $\mathfrak{i}=10$ sind demnach die Feldströme im Raumladungsgebiet sehr groß, verglichen mit den Feldströmen im Bahngebiet und damit nach (10) auch mit dem Gesamtstrom; es muß also in der Raumladungszone praktisch Boltzmann-Gleichgewicht herrschen. Da nun mit sinkender Stromdichte diese Verhältnisse sich immer günstiger in dem hier besprochenen Sinne einer Annäherung an ein BoLtzmanN-Gleichgewicht gestalten (man vgl. Abb. 9), wird man also erst recht auch bei kleineren Werten von $\dot{i}$ mit guter Berechtigung den Potentialabfall über dem Raumladungsgebiet durch (61) ausdrücken können. Die Gl. (59) muß daher auch in dem Übergangsgebiet $\mathrm{i} \approx 1$ gelten.

21 Der Spannungsabfall über den Bahngebieten ist ebenso wie in (16) vernachlässigt. Die Berechtigung dazu ergibt sich z. B. aus Abb. 12. 
Herrn Dr. E. Spenke bin ich für die Anregung zu dieser Untersuchung, für Diskussionen und Mithilfe bei der Gestaltung der Arbeit zu Dank verpflichtet. Insbesondere an der Darstellung von Abschnitt II, in dem die Raumladungszone behandelt wird, hat Herr Spenke wesentlichen Anteil.

\section{Nachtrag: Die Raumladungszone}

\section{bei kleinen und mittleren Stromdichten ${ }^{22}$}

Die am Schluß von III gewonnene Erkenntnis, $\mathrm{da} ß$ in der Raumladungszone noch bei verhältnismäßig hohen Stromdichten Boltzmann-Gleichgewicht herrscht, erlaubt uns, die Lösung für das Raumladungsgebiet auch für mittlere und kleine Stromdichten zu entwickeln. Benutzen wir die in Gl. (37) eingeführte Größe

$$
\varphi(x)=\frac{V(x)-V(0)}{\mathfrak{B}}
$$

und berücksichtigen wir (41), so schreibt sich der für Boltzmann-Gleichgewichte typische ${ }^{23}$ exponentielle Zusammenhang zwischen Konzentration und Potential folgendermaßen:

$$
\begin{aligned}
& p(x)=p(0) e^{-\varphi(x)}, \\
& n(x)=p(0) e^{+\varphi(x)} .
\end{aligned}
$$

Setzen wir diese Beziehungen in die Porssonsche Gl. (34) ein, so ergibt sich mit nochmaliger Berücksichtigung von (62)

$$
\frac{\mathrm{d}^{2} \varphi}{\mathrm{d} x^{2}}=\frac{4 \pi e n_{\mathrm{A}^{-}}}{\varepsilon \mathfrak{B}}\left\{\frac{2 p(0)}{n_{\mathrm{A}^{-}}} \sigma_{\text {int }} \varphi+1\right\} .
$$

Aus der Definitionsgleichung (62) folgt unmittelbar, da $\beta \varphi(x)$ neben dieser Differentialgleichung noch die Randbedingung

$$
\varphi(0)=0
$$

erfüllen muß. Zur vollständigen Festlegung der Lösung brauchen wir aber noch eine zweite Nebenbedingung, und als solche wählen wir

$$
\frac{\mathrm{d} \varphi}{\mathrm{d} x}=0 \quad \text { für } \quad x=x_{\mathrm{p}}=-\infty .
$$

Die Bahnfeldstärke ist nämlich bis zu mittleren Strombelastungen hinaus sehr klein gegenüber den in der Raumladungszone vorhandenen Potential-

22 Die folgenden Überlegungen und Ergebnisse hat mir Herr Dr. E. SPENKE zur Verfügung gestellt. Die numerischen gradienten (betragsmäßig!), so daß das physikalisch Wesentliche mit dem Nullsetzen der Neigung $\mathrm{d} \varphi / \mathrm{d} x$ am Rand $x_{\mathrm{p}}$ der Raumladungszone erfaßt wird. Daß man diese Grenze $x_{\mathrm{p}}$ der Raumladungszone formal wieder nach $-\infty$ verlegt, hat die schon am Anfang von Abschnitt II dargelegten Gründe.

Die Lösung von (65), (66) und (67) ist

$$
\begin{aligned}
& x=\sqrt{\frac{4 \pi e n_{A^{-}}}{\varepsilon \mathfrak{B}}} \\
& \cdot \int_{\varphi=0}^{\varphi} \frac{\mathrm{d} \varphi}{\sqrt{2\left[\varphi-\varphi\left(x_{\mathrm{p}}\right)\right]+\frac{4 p(0)}{n_{\mathrm{A}^{-}}}}\left[\operatorname{Eoj} \varphi-\operatorname{Co}\left\lceil\varphi\left(x_{\mathrm{p}}\right)\right]\right.}
\end{aligned}
$$

In diesem Ausdruck müssen noch $\varphi\left(x_{\mathrm{p}}\right)$ und $p(0)$ als Funktionen der Strombelastung $i$ eingesetzt werden. Hierfür können wir allerdings die Gln. (46) und (52), die nur für hohe i-Werte gelten, nicht übernehmen. Aber die Gleichungen, die bei der Annahme von Boltzmann-Gleichgewicht an die Stelle von (46) und (52) treten, sind sofort ermittelt: Multiplikation von (63) und (64), Anwendung des

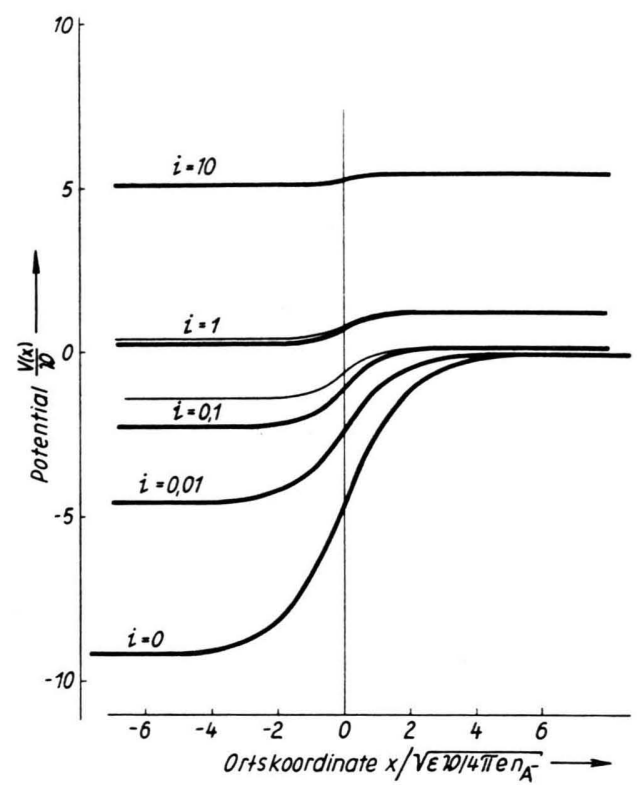

Abb. 13. Potentialverlauf in der Raumladungszone bei niedrigen und hohen Injektionen. (Ortskoordinate gegenüber Abb. 11 um mehrere Größenordnungen gedehnt!) —— Verlauf nach Gl. (68), - Verlauf nach Gl. (47).

Auswertungen erfolgten im Mathematischen Institut der Siemens-Schuckertwerke AG., Erlangen.

${ }^{23}$ Siehe $^{3}$, Gln. IV 2.07, IV 6.10 und IV 6.11. 
Resultats auf die Stelle $x=x_{\mathrm{p}}$ und Benutzung von (27) und (28) liefert

$$
\begin{aligned}
p(0) & =\sqrt{p\left(x_{\mathrm{p}}\right) \cdot n\left(x_{\mathrm{p}}\right)} \\
& =n_{\mathrm{A}^{-}} \cdot \sqrt{\mathrm{i}+\left(\frac{n_{\mathrm{i}}}{n_{\mathrm{A}^{-}}}\right)^{2}\left[\sqrt{\mathrm{i}+\frac{1}{4}}+\frac{1}{2}\right] .}
\end{aligned}
$$

während Division von (64) und (63), Anwendung auf die Stelle $x=x_{\mathrm{p}}$ und Benutzung von (27) und (28)

$$
\varphi\left(x_{\mathrm{p}}\right)=\frac{1}{2} \ln \frac{n\left(x_{\mathrm{p}}\right)}{p\left(x_{\mathrm{p}}\right)}=\frac{1}{2} \ln \frac{\sqrt{\mathrm{i}+\frac{1}{4}-\frac{1}{2}+\left(\begin{array}{c}
n_{\mathrm{i}} \\
n_{\mathrm{A}^{-}}
\end{array}\right)^{2}}}{\sqrt{\mathrm{i}+\frac{1}{4}+\frac{1}{2}}}
$$

ergibt, eine Beziehung, die wir implizit schon in $\mathrm{Ab}$ schnitt III benutzt haben [man vgl. Gln. (59) und (61) ].
Die Gln. (68), (69) und (70) beschreiben zusammen mit (63) und (64) die Lösung in der Raumladungszone für kleine und mittlere Stromdichten vollständig. Die Integration in (68) $\mathrm{mu} \beta$ dabei numerisch oder graphisch vorgenommen werden. Als Beispiel für die Auswertung ist in Abb. 13 der Potentialverlauf in der Raumladungszone für die Stromwerte der Abb. 11 aufgetragen. Für $i=0,1$ und $i=1$ ist die Lösung nach Gl. (47) mit eingezeichnet. Sie ist bei diesen relativ kleinen Strombelastungen noch keine gute Näherung. Das ändert sich aber bei größeren Strombelastungen. Für $i=10$ stimmt die Lösung (47) mit (68) innerhalb der zeichnerischen Genauigkeit überein.

Ab $\dot{i}=1000$ beginnt dann die Lösung (68) zu versagen. Wie man aus Abb. 9 ersieht, wird dann nämlich die in BoLtzmann-Lösung vernachlässigte Bahnfeldstärke wesentlich.

\section{NOTIZEN}

\section{Über die elektrischen Eigenschaften von InAs III}

\author{
Von O. G. Folberth und H. Weiss
}

Forschungslaboratorium der Siemens-Schuckertwerke AG., Erlangen

(Z. Naturforschg. 11 a, $510-511$ [1956]; eingegangen am 10. März 1956)

Herrn Professor Trendelenburg zum 60. Geburtstag gewidmet

In den beiden vorausgegangenen Arbeiten über die elektrischen Eigenschaften von $\operatorname{InAs}{ }^{1,2}$ wurde über Messungen an verhältnismäßig stark dotierten Proben berichtet. Die reinste n-leitende Probe besaß etwa $n_{\mathrm{d}} \approx 1,2 \cdot 10^{15}$ Donatoren $/ \mathrm{cm}^{3}$, die reinste p-leitende etwa $n_{\mathrm{A}} \approx 2 \cdot 10^{17}$ Akzeptoren $/ \mathrm{cm}^{3}$. Die vorliegende Arbeit beschäftigt sich nun mit schwächer dotierten p-leitenden Proben $\left(n_{\mathrm{A}}<2 \cdot 10^{17} \mathrm{~cm}^{-3}\right)$. Diese zeigen gegenüber den bisher bekannten Erscheinungen anomale Eigenschaften der elektrischen Leitfähigkeit und des HaLL-Effektes.

Die polykristallinen Präparate wurden nach bereits beschriebenen ${ }^{2}$ Methoden hergestellt und untersucht. Als Ausgangsmaterial diente n-leitendes InAs mit $n_{\mathrm{d}} \approx 3 \cdot 10^{16} \mathrm{~cm}^{-3}$. Probe 4 wurde mit $2 \cdot 10^{17}$ Zinkatomen $/ \mathrm{cm}^{3}$ dotiert. Sie entspricht etwa der Probe 1 in ${ }^{2}$ und verhält sich normal (Abb. 1). Präparat 3 enthält nur die halbe Menge an Akzeptoren. Entsprechend dem

1 O. G. Folberth, R. Grimm u. H. Weiss, Z. Naturforschg. 8 a. 826 [1953].

2 O. G. Folberth, O. Madelung u. H. Weiss, Z. Naturforschg. 9 a, 954 [1954].
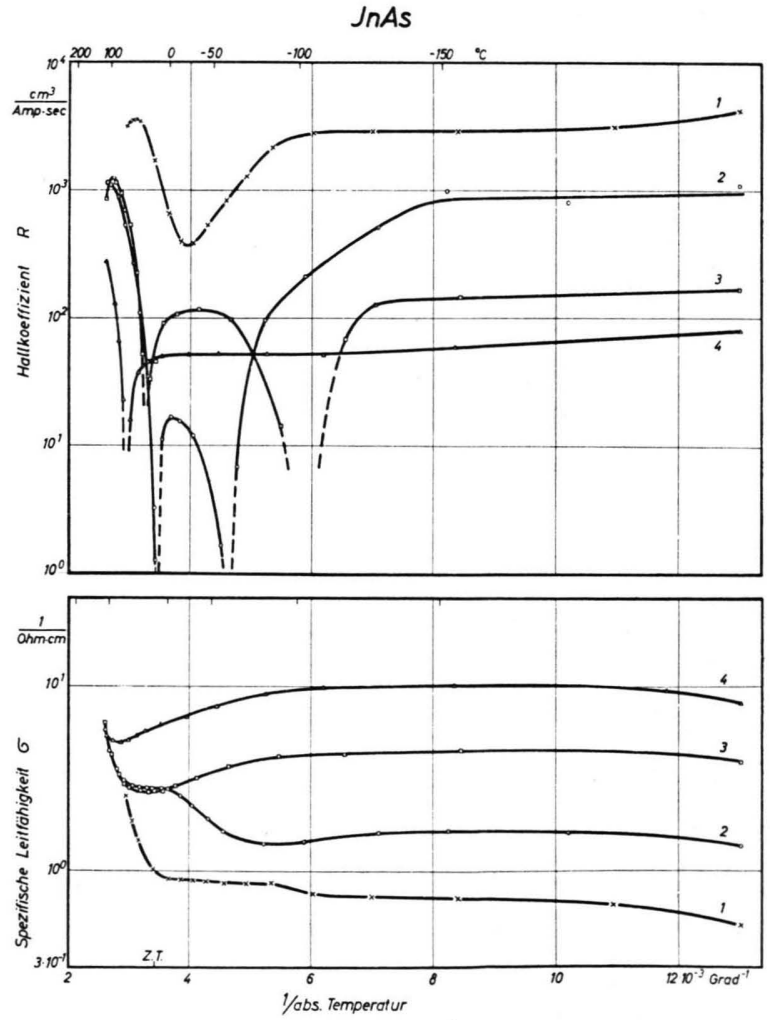

Abb. 1. Spezifische Leitfähigkeit und Hall-Koeffizient von $\mathrm{Zn}$-dotierten Proben InAs in Abhängigkeit von $1 / T$. 